\title{
The Automatic Baire Property and an Effective Property of $\omega$-Rational Functions
}

\author{
Olivier Finkel ${ }^{(\otimes)}(\mathbb{D}$ \\ Institut de Mathématiques de Jussieu - Paris Rive Gauche, \\ CNRS et Université Paris 7, Paris, France \\ Olivier.Finkel@math.univ-paris-diderot.fr
}

\begin{abstract}
We prove that $\omega$-regular languages accepted by Büchi or Muller automata satisfy an effective automata-theoretic version of the Baire property. Then we use this result to obtain a new effective property of rational functions over infinite words which are realized by finite state Büchi transducers: for each such function $F: \Sigma^{\omega} \rightarrow \Gamma^{\omega}$, one can construct a deterministic Büchi automaton $\mathcal{A}$ accepting a dense $\boldsymbol{\Pi}_{2}^{0}$-subset of $\Sigma^{\omega}$ such that the restriction of $F$ to $L(\mathcal{A})$ is continuous.
\end{abstract}

Keywords: Decision problems · Regular languages of infinite words • Infinitary rational relations $\cdot \omega$-rational functions $\cdot$ Topology $\cdot$ Automatic Baire property · Points of continuity

\section{Introduction}

Infinitary rational relations were first studied by Gire and Nivat $[8,10]$. The $\omega$ rational functions over infinite words, whose graphs are (functional) infinitary rational relations accepted by 2 -tape Büchi automata, have been studied by several authors $[1,4,16,18]$.

In this paper we are mainly interested in the question of the continuity of such $\omega$-rational functions. Recall that Prieur proved that one can decide whether a given $\omega$-rational function is continuous $[16,17]$. On the other hand, Carton, Finkel and Simonnet proved that one cannot decide whether a given $\omega$-rational function $f$ has at least one point of continuity [3]. Notice that this decision problem is actually $\Sigma_{1}^{1}$-complete, hence highly undecidable [6]. It was also proved in [3] that one cannot decide whether the continuity set of a given $\omega$-rational function $f$ (its set of continuity points) is a regular (respectively, context-free) $\omega$-language. Notice that the situation was shown to be quite different in the case of synchronous functions. It was proved in [3] that if $f: A^{\omega} \rightarrow B^{\omega}$ is an $\omega$-rational synchronous function, then the continuity set $C(f)$ of $f$ is $\omega$-rational. Moreover, if $X$ is an $\omega$-rational $\Pi_{2}^{0}$ subset of $A^{\omega}$, then $X$ is the continuity set $C(f)$ of some rational synchronous function $f$ of domain $A^{\omega}$. Notice that these previous works on the continuity of $\omega$-rational functions had shown that decision 
problems in this area may be decidable or not, while it is well known that most problems about regular languages accepted by finite automata are decidable.

We establish in this paper a new effective property of rational functions over infinite words. We first prove that $\omega$-regular languages accepted by Büchi or Muller automata satisfy an effective automata-theoretic version of the Baire property. Then we use this result to obtain a new effective property of rational functions over infinite words which are realized by finite state Büchi transducers: for each such function $F: \Sigma^{\omega} \rightarrow \Gamma^{\omega}$, one can construct a deterministic Büchi automaton $\mathcal{A}$ accepting a dense $\boldsymbol{\Pi}_{2}^{0}$-subset of $\Sigma^{\omega}$ such that the restriction of $F$ to this dense set $L(\mathcal{A})$ is continuous.

The paper is organized as follows. We recall basic notions on automata and on the Borel hierarchy in Sect.2. The automatic Baire property for regular $\omega$ languages is proved in Sect. 3. We prove our main new result on $\omega$-rational functions in Sect. 4. Some concluding remarks are given in Sect. 5 .

\section{Recall of Basic Notions}

We assume the reader to be familiar with the theory of formal $(\omega)$-languages $[18,20]$. We recall some usual notations of formal language theory.

When $\Sigma$ is a finite alphabet, a non-empty finite word over $\Sigma$ is any sequence $x=a_{1} \ldots a_{k}$, where $a_{i} \in \Sigma$ for $i=1, \ldots, k$, and $k$ is an integer $\geq 1$. The length of $x$ is $|x|=k$. The set of finite words (including the empty word $\varepsilon$ whose length is zero) over $\Sigma$ is denoted $\Sigma^{\star}$.

The first infinite ordinal is $\omega$. An $\omega$-word over $\Sigma$ is an $\omega$-sequence $a_{1} \ldots a_{n} \ldots$, where for all integers $i \geq 1, a_{i} \in \Sigma$. When $\sigma$ is an $\omega$-word over $\Sigma$, we write $\sigma=\sigma(1) \sigma(2) \ldots \sigma(n) \ldots$, where for all $i, \sigma(i) \in \Sigma$, and $\sigma[n]=\sigma(1) \sigma(2) \ldots \sigma(n)$.

The usual concatenation product of two finite words $u$ and $v$ is denoted $u \cdot v$ and sometimes just $u v$. This product is extended to the product of a finite word $u$ and an $\omega$-word $v$. The infinite word $u \cdot v$ is then the $\omega$-word such that: $(u \cdot v)(k)=$ $u(k)$ if $k \leq|u|$, and $(u \cdot v)(k)=v(k-|u|)$ if $k>|u|$. The concatenation product can be extended in an obvious way to the concatenation of an infinite sequence of finite words. The concatenation of a set $U$ of finite words with a set $V$ of infinite words is the set of infinite words $U \cdot V=\{u \cdot v \mid u \in U$ and $v \in V\}$. If $u$ is a finite word and $V$ is a set of infinite words then $u \cdot V=\{u \cdot v \mid v \in V\}$.

The set of $\omega$-words over the alphabet $\Sigma$ is denoted by $\Sigma^{\omega}$. An $\omega$-language over an alphabet $\Sigma$ is a subset of $\Sigma^{\omega}$.

Definition 1. A finite state machine (FSM) is a quadruple $\mathcal{M}=\left(K, \Sigma, \delta, q_{0}\right)$, where $K$ is a finite set of states, $\Sigma$ is a finite input alphabet, $q_{0} \in K$ is the initial state and $\delta$ is a mapping from $K \times \Sigma$ into $2^{K}$. A FSM is called deterministic iff: $\delta: K \times \Sigma \rightarrow\{\{q\} \mid q \in K\}$. (As usual, by a clear identification, we might consider in that case that $\delta: K \times \Sigma \rightarrow K)$.

$A$ Büchi automaton (BA) is a 5-tuple $\mathcal{A}=\left(K, \Sigma, \delta, q_{0}, F\right)$ where $\mathcal{M}=$ $\left(K, \Sigma, \delta, q_{0}\right)$ is a finite state machine and $F \subseteq K$ is the set of final states.

A Muller automaton (MA) is a 5-tuple $\mathcal{A}=\left(K, \Sigma, \delta, q_{0}, \mathcal{F}\right)$ where $\mathcal{M}=$ $\left(K, \Sigma, \delta, q_{0}\right)$ is a FSM and $\mathcal{F} \subseteq 2^{K}$ is the collection of designated state sets. 
A Büchi or Muller automaton is said to be deterministic if the associated FSM is deterministic.

Let $\sigma=a_{1} a_{2} \ldots a_{n} \ldots$ be an $\omega$-word over $\Sigma$.

A sequence of states $r=q_{1} q_{2} \ldots q_{n} \ldots$ is called an (infinite) run of $\mathcal{M}=$ $\left(K, \Sigma, \delta, q_{0}\right)$ on $\sigma$, starting in state $p$, iff: 1) $q_{1}=p$ and 2) for each $i \geq 1$, $q_{i+1} \in \delta\left(q_{i}, a_{i}\right)$.

In case a run $r$ of $\mathcal{M}$ on $\sigma$ starts in state $q_{0}$, we call it simply "a run of $\mathcal{M}$ on $\sigma$ ". For every (infinite) run $r=q_{1} q_{2} \ldots q_{n} \ldots$ of $\mathcal{M}, \operatorname{In}(\mathrm{r})$ is the set of states in $K$ entered by $\mathcal{M}$ infinitely many times during run $r: \operatorname{In}(\mathrm{r})=\left\{\mathrm{q} \in \mathrm{K} \mid \exists^{\infty} \mathrm{i} \geq\right.$ $\left.1 \mathrm{q}_{\mathrm{i}}=\mathrm{q}\right\}$.

For $\mathcal{A}=\left(K, \Sigma, \delta, q_{0}, F\right)$ a $B A$, the $\omega$-language accepted by $\mathcal{A}$ is:

$L(\mathcal{A})=\left\{\sigma \in \Sigma^{\omega} \mid\right.$ there exists a run $r$ of $\mathcal{A}$ on $\sigma$ such that $\left.\operatorname{In}(\mathrm{r}) \cap \mathrm{F} \neq \emptyset\right\}$.

For $\mathcal{A}=\left(K, \Sigma, \delta, q_{0}, F\right)$ a $M A$, the $\omega$-language accepted by $\mathcal{A}$ is:

$L(\mathcal{A})=\left\{\sigma \in \Sigma^{\omega} \mid\right.$ there exists a run $r$ of $\mathcal{A}$ on $\sigma$ such that $\left.\operatorname{In}(\mathrm{r}) \in \mathcal{F}\right\}$.

By R. Mc Naughton's Theorem, see [15], the expressive power of deterministic MA (DMA) is equal to the expressive power of non deterministic MA which is also equal to the expressive power of non deterministic BA.

Theorem 2. For any $\omega$-language $L \subseteq \Sigma^{\omega}$, the following conditions are equivalent:

1. There exists a DMA that accepts $L$.

2. There exists a MA that accepts $L$.

3. There exists a BA that accepts $L$.

An $\omega$-language $L$ satisfying one of these conditions is called a regular $\omega$-language.

Recall that, from a Büchi (respectively, Muller) automaton $\mathcal{A}$, one can effectively construct a deterministic Muller (respectively, non-deterministic Büchi) automaton $\mathcal{B}$ such that $L(\mathcal{A})=L(\mathcal{B})$.

A way to study the complexity of $\omega$-languages accepted by various automata is to study their topological complexity.

We assume the reader to be familiar with basic notions of topology which may be found in $[11,15,18]$. If $X$ is a finite alphabet containing at least two letters, then the set $X^{\omega}$ of infinite words over $X$ may be equipped with the product topology of the discrete topology on $X$. This topology is induced by a natural metric which is called the prefix metric and is defined as follows. For $u, v \in X^{\omega}$ and $u \neq v$ let $\delta(u, v)=2^{-l_{\operatorname{pref}(u, v)}}$ where $l_{\text {pref }(u, v)}$ is the first integer $n$ such that $u(n+1)$ is different from $v(n+1)$. The topological space $X^{\omega}$ is a Cantor space. The open sets of $X^{\omega}$ are the sets of the form $W \cdot X^{\omega}$, where $W \subseteq X^{\star}$. A set $L \subseteq X^{\omega}$ is a closed set iff its complement $X^{\omega}-L$ is an open set. Closed sets are characterized by the following:

Proposition 3. A set $L \subseteq X^{\omega}$ is a closed set of $X^{\omega}$ iff for every $\sigma \in X^{\omega}$, $\left[\forall n \geq 1, \exists u \in X^{\omega}\right.$ such that $\left.\sigma[n] \cdot u \in L\right]$ implies that $\sigma \in L$.

Define now the next classes of the Borel hierarchy: 
Definition 4. The classes $\boldsymbol{\Sigma}_{n}^{0}$ and $\boldsymbol{\Pi}_{n}^{0}$ of the Borel Hierarchy on the topological space $X^{\omega}$ are defined as follows: $\boldsymbol{\Sigma}_{1}^{0}$ is the class of open sets of $X^{\omega}, \Pi_{1}^{0}$ is the class of closed sets of $X^{\omega}$. And for any integer $n \geq 1: \boldsymbol{\Sigma}_{n+1}^{0}$ is the class of countable unions of $\boldsymbol{\Pi}_{n}^{0}$-subsets of $X^{\omega}$, and $\boldsymbol{\Pi}_{n+1}^{0}$ is the class of countable intersections of $\boldsymbol{\Sigma}_{n}^{0}$-subsets of $X^{\omega}$.

Remark 5. The hierarchy defined above is the hierarchy of Borel sets of finite rank. The Borel Hierarchy is also defined for transfinite levels (see [11]) but we shall not need this in the sequel. Recall that the class of Borel subsets of a Cantor space is the closure of the class of open sets under countable unions and countable intersections.

It turns out that there is a characterization of $\Pi_{2}^{0}$-subsets of $X^{\omega}$, involving the notion of $W^{\delta}$ which we now recall, see $[15,18]$.

Definition 6. For $W \subseteq X^{\star}$, we set: $W^{\delta}=\left\{\sigma \in X^{\omega} \mid \exists^{\infty} i\right.$ such that $\left.\sigma[i] \in W\right\}$. $\left(\sigma \in W^{\delta}\right.$ iff $\sigma$ has infinitely many prefixes in $W$.)

Then we can state the following proposition.

Proposition 7. A subset $L$ of $X^{\omega}$ is a $\Pi_{2}^{0}$-subset of $X^{\omega}$ iff there exists a set $W \subseteq X^{\star}$ such that $L=W^{\delta}$.

It is easy to see, using the above characterization of $\Pi_{2}^{0}$-sets, that every $\omega$ language accepted by a deterministic Büchi automaton is a $\boldsymbol{\Pi}_{2}^{0}$-set. Thus every regular $\omega$-language is a finite Boolean combination of $\boldsymbol{\Pi}_{2}^{0}$-sets, because it is accepted by a deterministic Muller automaton and this implies that it is a finite boolean combination of $\omega$-languages accepted by deterministic Büchi automata.

Landweber studied the topological properties of regular $\omega$-languages in [13]. He characterized the regular $\omega$-languages in each of the Borel classes $\boldsymbol{\Sigma}_{1}^{0}, \boldsymbol{\Pi}_{1}^{0}$, $\boldsymbol{\Sigma}_{2}^{0}, \boldsymbol{\Pi}_{2}^{0}$, and showed that one can decide, for an effectively given regular $\boldsymbol{\omega}$ language $L$, whether $L$ is in $\Sigma_{1}^{0}, \Pi_{1}^{0}, \Sigma_{2}^{0}$, or $\boldsymbol{\Pi}_{2}^{0}$. In particular, it turned out that a regular $\omega$-language is in the class $\Pi_{2}^{0}$ iff it is accepted by a deterministic Büchi automaton.

Recall that, from a Büchi or Muller automaton $\mathcal{A}$, one can construct some Büchi or Muller automata $\mathcal{B}$ and $\mathcal{C}$, such that $L(\mathcal{B})$ is equal to the topological closure of $L(\mathcal{A})$, and $L(\mathcal{C})$ is equal to the topological interior of $L(\mathcal{A})$, see $[15,18]$.

\section{The Automatic Baire Property}

In this section we are going to prove an automatic version of the result stating that every Borel (and even every analytic) set has the Baire property.

We firstly recall some basic definitions about meager sets, see [11]. In a topological space $\mathcal{X}$, a set $A \subseteq \mathcal{X}$ is said to be nowhere dense if its closure $\bar{A}$ has empty interior, i.e. $\operatorname{Int}(\bar{A})=\emptyset$. A set $A \subseteq \mathcal{X}$ is said to be meager if it is the union of countably many nowhere dense sets, or equivalently if it is included in 
a countable union of closed sets with empty interiors. This means that $A$ is meager if there exist countably many closed sets $A_{n}, n \geq 1$, such that $A \subseteq \bigcup_{n \geq 1} A_{n}$ where for every integer $n \geq 1$, $\operatorname{Int}\left(A_{n}\right)=\emptyset$. A set is comeager if its complement is meager, i.e. if it contains the intersection of countably many dense open sets. Notice that the notion of a meager set is a notion of a small set, while the notion of a comeager set is a notion of a big set.

Recall that a Baire space is a topological space $\mathcal{X}$ in which every intersection of countably many dense open sets is dense, or equivalently in which every countable union of closed sets with empty interiors has also an empty interior. It is well known that every Cantor space $\Sigma^{\omega}$ is a Baire space. In the sequel we will consider only Cantor spaces.

We now recall the notion of Baire property. For any sets $A, B \subseteq \Sigma^{\omega}$, we denote by $A \Delta B$ the symmetric difference of $A$ and $B$, and we write $A={ }^{\star} B$ if and only if $A \Delta B$ is meager.

Definition 8. A set $A \subseteq \Sigma^{\omega}$ has the Baire property (BP) if there exists an open set $U \subseteq \Sigma^{\omega}$ such that $A=^{\star} U$.

An important result of descriptive set theory is the following result, see [11, page 47].

Theorem 9. Every Borel set of a Cantor space has the Baire property.

We are going to prove an automatic version of the above theorem. We first give the following definition.

Definition 10. Let $L=L(\mathcal{A}) \subseteq \Sigma^{\omega}$ be a regular $\omega$-language accepted by a Büchi or Muller automaton $\mathcal{A}$. The $\omega$-language $L$ is said to have the automatic Baire property if one can construct from $\mathcal{A}$ some Büchi automata $\mathcal{B}$ and $\mathcal{C}$ such that $L(\mathcal{B}) \subseteq \Sigma^{\omega}$ is open, $L(\mathcal{C}) \subseteq \Sigma^{\omega}$ is a countable union of closed sets with empty interior, i.e. a meager $\Sigma_{2}^{0}$-set, and $L(\mathcal{A}) \Delta L(\mathcal{B}) \subseteq L(\mathcal{C})$.

We already know that the regular $\omega$-languages have the Baire property since they are Borel. We now state the following theorem which gives an automatic version of this result.

Theorem 11. Let $L=L(\mathcal{A}) \subseteq \Sigma^{\omega}$ be a regular $\omega$-language accepted by a Büchi or Muller automaton $\mathcal{A}$. Then one can construct Büchi automata $\mathcal{B}$ and $\mathcal{C}$ such that $L(\mathcal{B}) \subseteq \Sigma^{\omega}$ is open, $L(\mathcal{C}) \subseteq \Sigma^{\omega}$ is a meager $\Sigma_{2}^{0}$-set, and $L(\mathcal{A}) \Delta L(\mathcal{B}) \subseteq L(\mathcal{C})$, i.e. the $\omega$-language $L(\mathcal{A})$ has the automatic Baire property.

In order to prove this result, we first prove the following lemmas.

Lemma 12. Every regular $\omega$-language which is open or closed has the automatic Baire property.

Proof. Let $L=L(\mathcal{A}) \subseteq \Sigma^{\omega}$ be a regular $\omega$-language accepted by a Büchi or Muller automaton $\mathcal{A}$. 
If $L=L(\mathcal{A})$ is an open set then we immediately see that we get the result with $\mathcal{B}=\mathcal{A}$ and $\mathcal{C}$ is any Büchi automaton accepting the empty set.

If $L=L(\mathcal{A})$ is a closed set then $L \backslash \operatorname{Int}(L)$ is a closed set with empty interior. Moreover it is known that one can construct from the Büchi automaton $\mathcal{A}$ another Büchi automaton $\mathcal{B}$ accepting $\operatorname{Int}(L)$, and then also a Büchi automaton $\mathcal{C}$ accepting $L \backslash \operatorname{Int}(L)$. Then we have $L(\mathcal{A}) \Delta L(\mathcal{B})=L \backslash \operatorname{Int}(L)=L(\mathcal{C})$, with $L(\mathcal{B})$ open and $L(\mathcal{C})$ is a closed set with empty interior.

Lemma 13. Every regular $\omega$-language which is a $\boldsymbol{\Sigma}_{2}^{0}$-set has the automatic Baire property.

Lemma 14. Let $L \subseteq \Sigma^{\omega}$ be a regular $\omega$-language which has the automatic Baire property. Then its complement $\Sigma^{\omega} \backslash L$ has also the automatic Baire property.

Lemma 15. The class of regular $\omega$-languages having the automatic Baire property is closed under finite union and under finite intersection.

End of Proof of Theorem 11. We now return to the general case of a regular $\omega$-language $L \subseteq \Sigma^{\omega}$, accepted by a Büchi or Muller automaton. We know that we can construct a deterministic Muller automaton $\mathcal{A}=\left(K, \Sigma, \delta, q_{0}, \mathcal{F}\right)$ accepting $L$. Recall that $\mathcal{F} \subseteq 2^{K}$ is here the collection of designated state sets. For each state $q \in K$, we now denote by $\mathcal{A}^{(q)}$ the automaton $\mathcal{A}$ but viewed as a (deterministic) Büchi automaton with the single accepting state $q$, i.e. $\mathcal{A}^{(q)}=\left(K, \Sigma, \delta, q_{0},\{q\}\right)$. We know that the languages $L\left(\mathcal{A}^{(q)}\right)$ are Borel $\boldsymbol{\Pi}_{2}^{0}$-sets and thus satisfy the automatic Baire property by Lemmas 13 and 14. Moreover we have the following equality:

$$
L(\mathcal{A})=\bigcup_{F \in \mathcal{F}}\left[\bigcap_{q \in F} L\left(\mathcal{A}^{(q)}\right) \backslash \bigcup_{q \notin F} L\left(\mathcal{A}^{(q)}\right)\right]
$$

This implies, from the previous lemmas about the preservation of the automatic Baire property by Boolean operations, that we can construct Büchi automata $\mathcal{B}$ and $\mathcal{C}$, such that $L(\mathcal{B})$ is open and $L(\mathcal{C})$ is a meager $\boldsymbol{\Sigma}_{2}^{0}$-set, which satisfy $L(\mathcal{A}) \Delta L(\mathcal{B}) \subseteq L(\mathcal{C})$. Thus the $\omega$-language $L$ has the automatic Baire property.

Corollary 16. On can decide, for a given Büchi or Muller automaton $\mathcal{A}$, whether $L(\mathcal{A})$ is meager.

Proof. Let $\mathcal{A}$ be a Büchi or Muller automaton. The $\omega$-language $L(\mathcal{A})$ has the automatic Baire property and we can construct Büchi automata $\mathcal{B}$ and $\mathcal{C}$, such that $L(\mathcal{B})$ is open and $L(\mathcal{C})$ is a countable union of closed sets with empty interiors, which satisfy $L(\mathcal{A}) \Delta L(\mathcal{B}) \subseteq L(\mathcal{C})$. It is easy to see that $L(\mathcal{A})$ is meager if and only if $L(\mathcal{B})$ is empty, since any non-empty open set is non-meager, and it can be decided from the automaton $\mathcal{B}$ whether $L(\mathcal{B})$ is empty.

Remark 17. The above Corollary followed already from Staiger's paper [19], see also [14]. So we get here another proof of this result, based on the automatic Baire property. 


\section{An Application to $\omega$-Rational Functions}

\subsection{Infinitary Rational Relations}

We now recall the definition of infinitary rational relations, via definition by Büchi transducers:

Definition 18. A 2-tape Büchi automaton is a 6-tuple $\mathcal{T}=\left(K, \Sigma, \Gamma, \Delta, q_{0}, F\right)$, where $K$ is a finite set of states, $\Sigma$ and $\Gamma$ are finite sets called the input and the output alphabets, $\Delta$ is a finite subset of $K \times(\Sigma \cup \varepsilon) \times(\Gamma \cup \varepsilon) \times K$ called the set of transitions, $q_{0}$ is the initial state, and $F \subseteq K$ is the set of accepting states. $A$ computation $\mathcal{C}$ of the automaton $\mathcal{T}$ is an infinite sequence of consecutive transitions

$$
\left(q_{0}, u_{1}, v_{1}, q_{1}\right),\left(q_{1}, u_{2}, v_{2}, q_{2}\right), \ldots\left(q_{i-1}, u_{i}, v_{i}, q_{i}\right),\left(q_{i}, u_{i+1}, v_{i+1}, q_{i+1}\right), \ldots
$$

The computation is said to be successful iff there exists a final state $q_{f} \in F$ and infinitely many integers $i \geq 0$ such that $q_{i}=q_{f}$. The input word and output word of the computation are respectively $u=u_{1} \cdot u_{2} . u_{3} \ldots$ and $v=v_{1} \cdot v_{2} \cdot v_{3} \ldots$ The input and the output words may be finite or infinite. The infinitary rational relation $R(\mathcal{T}) \subseteq \Sigma^{\omega} \times \Gamma^{\omega}$ accepted by the 2-tape Büchi automaton $\mathcal{T}$ is the set of pairs $(u, v) \in \Sigma^{\omega} \times \Gamma^{\omega}$ such that $u$ and $v$ are the input and the output words of some successful computation $\mathcal{C}$ of $\mathcal{T}$.

The 2-tape Büchi automaton $\mathcal{T}=\left(K, \Sigma, \Gamma, \Delta, q_{0}, F\right)$ is said to be synchronous if the set of transitions $\Delta$ is a finite subset of $K \times \Sigma \times \Gamma \times K$, i.e. if each transition is labelled with a pair $(a, b) \in \Sigma \times \Gamma$. An infinitary rational relation recognized by a synchronous 2-tape Büchi automaton is in fact, via the natural identification of $\Sigma^{\omega} \times \Gamma^{\omega}$ with $(\Sigma \times \Gamma)^{\omega}$, an $\omega$-language over the product alphabet $\Sigma \times \Gamma$ which is accepted by a Büchi automaton. It is called a synchronous infinitary rational relation. An infinitary rational relation is said to be asynchronous if it can not be recognized by any synchronous 2-tape Büchi automaton.

Remark 19. In the above definition, we could have defined the set of transitions $\Delta$ as a subset of $K \times \Sigma^{\star} \times \Gamma^{\star} \times K$. We have chosen to define $\Delta$ as a finite subset of $K \times(\Sigma \cup \varepsilon) \times(\Gamma \cup \varepsilon) \times K$ to simplify the proofs. However this is done without loss of generality because it is easy to see that this convention does not change the class of infinitary rational relations.

If $R(\mathcal{T}) \subseteq \Sigma^{\omega} \times \Gamma^{\omega}$ is an infinitary rational relation recognized by the 2 -tape Büchi automaton $\mathcal{T}$ then we denote

$$
\operatorname{Dom}(R(\mathcal{T}))=\left\{u \in \Sigma^{\omega} \mid \exists v \in \Gamma^{\omega} \quad(u, v) \in R(\mathcal{T})\right\}
$$

and

$$
\operatorname{Im}(R(\mathcal{T}))=\left\{v \in \Gamma^{\omega} \mid \exists u \in \Sigma^{\omega}(u, v) \in R(\mathcal{T})\right\} .
$$

It is well known that, for each infinitary rational relation $R(\mathcal{T}) \subseteq \Sigma^{\omega} \times \Gamma^{\omega}$, the sets $\operatorname{Dom}(R(\mathcal{T}))$ and $\operatorname{Im}(R(\mathcal{T}))$ are regular $\omega$-languages and that one can construct, from the Büchi transducer $\mathcal{T}$, some (non-deterministic) Büchi automata $\mathcal{A}$ and $\mathcal{B}$ accepting the $\omega$-languages $\operatorname{Dom}(R(\mathcal{T}))$ and $\operatorname{Im}(R(\mathcal{T}))$.

Recall now the following undecidability result of Frougny and Sakarovitch. 
Theorem 20 ([7]). One cannot decide whether a given infinitary rational relation is synchronous.

We proved in [5] that many decision problems about infinitary rational relations are highly undecidable. In fact many of them, like the universality problem, the equivalence problem, the inclusion problem, the cofiniteness problem, the unambiguity problem, are $\Pi_{2}^{1}$-complete, hence located at the second level of the analytical hierarchy.

\subsection{Continuity of $\omega$-Rational Functions}

Recall that an infinitary rational relation $R(\mathcal{T}) \subseteq \Sigma^{\omega} \times \Gamma^{\omega}$ is said to be functional iff it is the graph of a function, i.e. iff

$$
[\forall x \in \operatorname{Dom}(R(\mathcal{T})) \quad \exists ! y \in \operatorname{Im}(R(\mathcal{T})) \quad(x, y) \in R(\mathcal{T})] .
$$

Then the functional relation $R(\mathcal{T})$ defines an $\omega$-rational (partial) function $F_{\mathcal{T}}$ : $\operatorname{Dom}(R(\mathcal{T})) \rightarrow \Gamma^{\omega}$ by: for each $u \in \operatorname{Dom}(R(\mathcal{T})), F_{\mathcal{T}}(u)$ is the unique $v \in \Gamma^{\omega}$ such that $(u, v) \in R(\mathcal{T})$.

An $\omega$-rational (partial) function $f: \Sigma^{\omega} \rightarrow \Gamma^{\omega}$ is said to be synchronous if there is a synchronous 2-tape Büchi automaton $\mathcal{T}$ such that $f=F_{\mathcal{T}}$.

An $\omega$-rational (partial) function $f: \Sigma^{\omega} \rightarrow \Gamma^{\omega}$ is said to be asynchronous if there is no synchronous 2-tape Büchi automaton $\mathcal{T}$ such that $f=F_{\mathcal{T}}$.

Recall the following previous decidability result.

Theorem 21 (Gire [9]). One can decide whether an infinitary rational relation recognized by a given 2 -tape Büchi automaton $\mathcal{T}$ is a functional infinitary rational relation.

It is very natural to consider the notion of continuity for $\omega$-rational functions defined by 2 -tape Büchi automata.

We recall that a function $f: \operatorname{Dom}(f) \subseteq \Sigma^{\omega} \rightarrow \Gamma^{\omega}$, whose domain is $\operatorname{Dom}(f)$, is said to be continuous at point $x \in \operatorname{Dom}(f)$ if :

$$
\forall n \geq 1 \quad \exists k \geq 1 \quad \forall y \in \operatorname{Dom}(f) \quad\left[\delta(x, y)<2^{-k} \Rightarrow \delta(f(x), f(y))<2^{-n}\right]
$$

The continuity set $C(f)$ of the function $f$ is the set of points of continuity of $f$. Notice that the continuity set $C(f)$ of a function $f: \Sigma^{\omega} \rightarrow \Gamma^{\omega}$ is always a Borel $\Pi_{2}^{0}$-subset of $\Sigma^{\omega}$, see [3].

The function $f$ is said to be continuous if it is continuous at every point $x \in \operatorname{Dom}(f)$, i. e. if $C(f)=\operatorname{Dom}(f)$.

Prieur proved the following decidability result.

Theorem 22 (Prieur $[16,17])$. One can decide whether a given $\omega$-rational function is continuous.

On the other hand the following undecidability result was proved in [3]. 
Theorem 23 (see [3]). One cannot decide whether a given w-rational function $f$ has at least one point of continuity.

The exact complexity of this undecidable problem was given in [6]. It is $\Sigma_{1}^{1}$ complete to determine whether a given $\omega$-rational function $f$ has at least one point of continuity.

We now consider the continuity set of an $\omega$-rational function and its possible complexity. The following undecidability result was proved in [3].

Theorem 24 (see [3]). One cannot decide whether the continuity set of a given $\omega$-rational function $f$ is a regular (respectively, context-free) $\omega$-language.

The situation is quite different in the case of synchronous functions. The following results were proved in [3].

Theorem 25 ([3]). Let $f: A^{\omega} \rightarrow B^{\omega}$ be a rational synchronous function. The continuity set $C(f)$ of $f$ is rational.

Theorem 26 ([3]). Let $X$ be a rational $\Pi_{2}^{0}$ subset of $A^{\omega}$. Then $X$ is the continuity set $C(f)$ of some rational synchronous function $f$ of domain $A^{\omega}$.

We are now going to prove another effective result about $\omega$-rational functions.

We first recall the following result of descriptive set theory, in the particular case of Cantor spaces $\Sigma^{\omega}$ and $\Gamma^{\omega}$. A Borel function $f: \Sigma^{\omega} \rightarrow \Gamma^{\omega}$ is a function for which the inverse image of any Borel subset of $\Gamma^{\omega}$, or equivalently of any open set of $\Gamma^{\omega}$, is a Borel subset of $\Sigma^{\omega}$.

Theorem 27 (see Theorem 8.38 of [11]). Let $\Sigma$ and $\Gamma$ be two finite alphabets and $f: \Sigma^{\omega} \rightarrow \Gamma^{\omega}$ be a Borel function. Then there is a dense $\Pi_{2}^{0}$-subset $G$ of $\Sigma^{\omega}$ such that the restriction of $f$ to $G$ is continuous.

We now state an automatic version of this theorem.

Theorem 28. Let $\Sigma$ and $\Gamma$ be two finite alphabets and $f: \Sigma^{\omega} \rightarrow \Gamma^{\omega}$ be an $\omega$-rational function. Then one can construct, from a 2-tape Büchi automaton accepting the graph of the function $f$, a deterministic Büchi automaton accepting a dense $\Pi_{2}^{0}$-subset $G$ of $\Sigma^{\omega}$ such that the restriction of $f$ to $G$ is continuous.

Proof. Let $\Sigma$ and $\Gamma$ be two finite alphabets and $f: \Sigma^{\omega} \rightarrow \Gamma^{\omega}$ be an $\omega$-rational function whose graph is accepted by a 2-tape Büchi automaton $\mathcal{A}=\left(K, \Sigma, \Gamma, \Delta, q_{0}, F\right)$.

Notice that one can also consider the 2-tape automaton $\mathcal{A}$ reading pairs of finite words $(v, u) \in \Sigma^{\star} \times \Gamma^{\star}$. A partial computation of the 2-tape automaton $\mathcal{A}$ reading such a pair $(v, u)$ is simply a finite sequence of consecutive transitions

$$
\left(q_{0}, a_{1}, b_{1}, q_{1}\right),\left(q_{1}, a_{2}, b_{2}, q_{2}\right), \ldots\left(q_{i-1}, a_{i}, b_{i}, q_{i}\right),\left(q_{i}, a_{i+1}, b_{i+1}, q_{i+1}\right)
$$

such that $v=a_{1} a_{2} \ldots a_{i+1}$ and $u=b_{1} b_{2} \ldots b_{i+1}$. This computation ends in state $q_{i+1}$. 
We assume that we have an effective enumeration of the finite words over the alphabet $\Gamma$ given by $\left(u_{n}\right)_{n \geq 1}, u_{n} \in \Gamma^{\star}$. For $q \in K$ we also denote $\mathcal{A}_{q}$ the automaton $\mathcal{A}$ in which we have changed the initial state so that the initial state of $\mathcal{A}_{q}$ is $q$ instead of $q_{0}$.

Let us now consider the basic open set of the space $\Gamma^{\omega}$ given by $U_{n}=u_{n} \cdot \Gamma^{\omega}$. We first describe $f^{-1}\left(U_{n}\right)$. An $\omega$-word $x \in \Sigma^{\omega}$ belongs to the set $f^{-1}\left(U_{n}\right)$ iff $x$ can be written in the form $x=v \cdot y$ for some words $v \in \Sigma^{\star}$ and $y \in \Sigma^{\omega}$, and there is a partial computation of the automaton $\mathcal{A}$ reading $\left(v, u_{n}\right)$ for which $\mathcal{A}$ is in state $q$ after having read the initial pair $\left(v, u_{n}\right) \in \Sigma^{\star} \times \Gamma^{\star}$ (where the finite words $v$ and $u_{n}$ might have different lengths if the automaton $\mathcal{A}$ is not synchronous), and $y \in \operatorname{Dom}\left(R\left(\mathcal{A}_{q}\right)\right)$. Recall that $R\left(\mathcal{A}_{q}\right) \subseteq(\Sigma \times \Gamma)^{\omega}$ is an infinitary rational relation and that $\operatorname{Dom}\left(R\left(\mathcal{A}_{q}\right)\right)$ is then a regular $\omega$-language and that one can construct from $\mathcal{A}$ a deterministic Muller automaton accepting this $\omega$-language $\operatorname{Dom}\left(R\left(\mathcal{A}_{q}\right)\right)$ which will be denoted $L_{q}$. We also denote $T\left(u_{n}, q\right)$ the set of finite words $v$ over $\Sigma$ such that the automaton $\mathcal{A}$ may be in state $q$ after having read the initial pair $\left(v, u_{n}\right) \in \Sigma^{\star} \times \Gamma^{\star}$. Then the following equality holds:

$$
f^{-1}\left(U_{n}\right)=\bigcup_{q \in K} T\left(u_{n}, q\right) \cdot L_{q}
$$

We can now apply the automatic Baire property stated in the above Theorem 11 . Then for each regular $\omega$-language $L_{q}$, one can construct a deterministic Muller automaton accepting an open set $O_{q}$ and a deterministic Muller automaton accepting a countable union $W_{q}$ of closed sets with empty interiors, such that for each $q \in K$,

$$
L_{q} \Delta O_{q} \subseteq W_{q}
$$

Now we set

$$
V_{n}=\bigcup_{q \in K} T\left(u_{n}, q\right) \cdot O_{q} \quad \text { and } \quad F_{n}=\bigcup_{q \in K} T\left(u_{n}, q\right) \cdot W_{q}
$$

Notice that each set $T\left(u_{n}, q\right)$ is countable and that for each finite word $u \in$ $T\left(u_{n}, q\right)$ it is easy to see that the set $u \cdot O_{q}$ is open and that the set $u \cdot W_{q}$ is a countable union of closed sets with empty interiors. Thus it is easy to see that $V_{n}$ is open, and that $F_{n}$ is a countable union of closed sets with empty interiors. Moreover it is easy to see that $V_{n}$ and $F_{n}$ are regular $\omega$-languages since each set $T\left(u_{n}, q\right)$ is a regular language of finite words over the alphabet $\Sigma$. Moreover it holds that:

$$
f^{-1}\left(U_{n}\right) \Delta V_{n} \subseteq F_{n}
$$

We now prove that $F=\bigcup_{n \geq 1} F_{n}$ is itself a regular $\omega$-language. It holds that

$$
F=\bigcup_{n \geq 1} F_{n}=\bigcup_{n \geq 1} \bigcup_{q \in K} T\left(u_{n}, q\right) \cdot W_{q}=\bigcup_{q \in K} \bigcup_{n \geq 1} T\left(u_{n}, q\right) \cdot W_{q}
$$

Consider now the 2-tape automaton $\mathcal{B}_{q}$ which is like the 2-tape automaton $\mathcal{A}$ but reads only pairs of finite words in $\Sigma^{\star} \times \Gamma^{\star}$ and has the state $q$ as unique 
accepting state. Let then $\mathcal{C}_{q}$ be a finite automaton which reads only finite words over the alphabet $\Sigma$ and such that $L\left(\mathcal{C}_{q}\right)=\operatorname{Proj}_{\Sigma \star}\left(L\left(\mathcal{B}_{q}\right)\right)$ is the projection of the language $L\left(\mathcal{B}_{q}\right)$ on $\Sigma^{\star}$. We can construct, from the automaton $\mathcal{A}$, the automata $\mathcal{B}_{q}$ and $\mathcal{C}_{q}$ for each $q \in K$. Now it holds that:

$$
F=\bigcup_{n \geq 1} F_{n}=\bigcup_{q \in K} \bigcup_{n \geq 1} T\left(u_{n}, q\right) \cdot W_{q}=\bigcup_{q \in K} L\left(\mathcal{C}_{q}\right) \cdot W_{q}
$$

On the other hand, for each finite word $u \in \Sigma^{\star}$, the set $u \cdot W_{q}$ is a meager $\boldsymbol{\Sigma}_{2}^{0}$-set, since $W_{q}$ is a meager $\boldsymbol{\Sigma}_{2}^{0}$-set. Thus the set

$$
F=\bigcup_{q \in K} L\left(\mathcal{C}_{q}\right) \cdot W_{q}
$$

is also a countable union of closed sets with empty interiors, since $K$ is finite and each language $L\left(\mathcal{C}_{q}\right)$ is countable. Moreover the $\omega$-language $F$ is regular and we can construct, from the automata $\mathcal{C}_{q}$ and from the deterministic Muller automata accepting the $\omega$-languages $W_{q}$, a deterministic Muller automaton accepting $F$.

We can now set $G_{n}=\Sigma^{\omega} \backslash F_{n}$ and $G=\bigcap_{n \geq 1} G_{n}=\Sigma^{\omega} \backslash \bigcup_{n>1} F_{n}=\Sigma^{\omega} \backslash F$. Then $G$ is a countable intersection of dense open subsets of $\Sigma \omega$, hence also a dense $\Pi_{2}^{0}$-subset $G$ of $\Sigma^{\omega}$. Moreover we can construct a deterministic Muller automaton and even a deterministic Büchi automaton (since $G$ is a $\boldsymbol{\Pi}_{2}^{0}$-set, see $\left[15\right.$, page 41]) accepting $G$. We can now see that the restriction $f_{G}$ of the function $f$ to $G$ is continuous. This follows from the fact that the inverse image of every basic open set of $\Gamma^{\omega}$ by the function $f_{G}$ is an open subset of $G$ because for each integer $n \geq 1$, it holds that $f_{G}^{-1}\left(U_{n}\right)=f^{-1}\left(U_{n}\right) \cap G=V_{n} \cap G$.

Remark 29. The above dense $\boldsymbol{\Pi}_{2}^{0}$-subset $G$ of $\Sigma^{\omega}$ is comeager and thus Theorem 28 shows that one can construct a deterministic Büchi automaton accepting a "big" $\omega$-rational subset of $\Sigma^{\omega}$ on which the function $f$ is continuous.

\section{Concluding Remarks}

We have proved a new effective property of $\omega$-rational functions. We hope this property will be useful for further studies involving $\omega$-rational functions. For instance an $\omega$-automatic structure is defined via synchronous infinitary rational relations, see $[2,12]$. On the other hand, any (synchronous) infinitary rational relation is uniformizable by a (synchronous) $\omega$-rational function, see [4]. Thus we can expect that our result will be useful in particular in the study of $\omega$-automatic structures.

We also hope that the automatic Baire property will be useful in other studies involving regular $\omega$-languages like the study of infinite games specified by automata. 


\section{References}

1. Béal, M.P., Carton, O., Prieur, C., Sakarovitch, J.: Squaring transducers: an efficient procedure for deciding functionality and sequentiality. Theor. Comput. Sci. 292(1), 45-63 (2003)

2. Blumensath, A., Grädel, E.: Finite presentations of infinite structures: automata and interpretations. Theory Comput. Syst. 37(6), 641-674 (2004)

3. Carton, O., Finkel, O., Simonnet, P.: On the continuity set of an omega rational function. Theor. Inform. Appl. 42(1), 183-196 (2008)

4. Choffrut, C., Grigorieff, S.: Uniformization of rational relations. In: Karhumäki, J., Maurer, H.A., Paun, G., Rozenberg, G. (eds.) Jewels are Forever, Contributions on Theoretical Computer Science in Honor of Arto Salomaa, pp. 59-71. Springer, Heidelberg (1999). https://doi.org/10.1007/978-3-642-60207-8_6

5. Finkel, O.: Highly undecidable problems for infinite computations. RAIRO-Theor. Inform. Appl. 43(2), 339-364 (2009)

6. Finkel, O.: Three applications to rational relations of the high undecidability of the infinite Post correspondence problem in a regular $\omega$-language. Int. J. Found. Comput. Sci. 23(7), 1481-1498 (2012)

7. Frougny, C., Sakarovitch, J.: Synchronized rational relations of finite and infinite words. Theor. Comput. Sci. 108(1), 45-82 (1993)

8. Gire, F.: Relations rationnelles infinitaires. Ph.D. thesis, Université Paris VII (1981)

9. Gire, F.: Two decidability problems for infinite words. Inf. Process. Lett. 22(3), 135-140 (1986)

10. Gire, F., Nivat, M.: Relations rationnelles infinitaires. Calcolo XXI, 91-125 (1984)

11. Kechris, A.S.: Classical Descriptive Set Theory. Springer, New York (1995). https://doi.org/10.1007/978-1-4612-4190-4

12. Kuske, D., Lohrey, M.: First-order and counting theories of omega-automatic structures. J. Symb. Logic 73(1), 129-150 (2008)

13. Landweber, L.: Decision problems for $\omega$-automata. Math. Syst. Theory 3(4), 376384 (1969)

14. Michalewski, H., Mio, M., Skrzypczak, M.: Monadic second order logic with measure and category quantifiers. Logical Methods Comput. Sci. 14(2) (2018)

15. Perrin, D., Pin, J.E.: Infinite Words, Automata, Semigroups, Logic and Games, Pure and Applied Mathematics, vol. 141. Elsevier, Amsterdam (2004)

16. Prieur, C.: How to decide continuity of rational functions on infinite words. Theor. Comput. Sci. 250(1-2), 71-82 (2001)

17. Prieur, C.: How to decide continuity of rational functions on infinite words. Theor. Comput. Sci. 276(1-2), 445-447 (2002)

18. Staiger, L.: $\omega$-languages. In: Rozenberg, G., Salomaa, A. (eds.) Handbook of Formal Languages, vol. 3, pp. 339-387. Springer, Heidelberg (1997). https://doi.org/10. 1007/978-3-642-59126-6_6

19. Staiger, L.: Rich $\omega$-words and monadic second-order arithmetic. In: Nielsen, M., Thomas, W. (eds.) CSL 1997. LNCS, vol. 1414, pp. 478-490. Springer, Heidelberg (1998). https://doi.org/10.1007/BFb0028032

20. Thomas, W.: Automata on infinite objects. In: van Leeuwen, J. (ed.) Handbook of Theoretical Computer Science. Formal Models and Semantics, vol. B, pp. 135-191. Elsevier, Amsterdam (1990) 